\title{
Improved Monte Carlo Localization of Autonomous Robots through Simultaneous Estimation of Motion Model Parameters
}

\author{
Jörg Müller Christoph Gonsior $\quad$ Wolfram Burgard
}

\begin{abstract}
In recent years, there has been an increasing interest in autonomous navigation for lightweight flying robots. With regard to self-localization flying robots have several limitations compared to ground vehicles. Due to their limited payload flying vehicles possess only limited computational resources and are restricted to a few and lightweight sensors. Additionally the kinematics of flying robots is rather complex, which requires sophisticated motion models that are typically hard to calibrate. However, as the sensors provide only a limited amount of information, the motion models need to be highly accurate to reduce the potential increase of uncertainty caused by the movements of the vehicle. In this paper, we present a novel approach to simultaneous localization and estimation of motion model parameters and their adaptation in the context of a particle filter. To deal with sudden changes of parameters, our approach utilizes random sampling augmented by additional damping to avoid oscillations caused by the delayed detection of the changes. As we demonstrate in experiments with a real blimp, our method can deal with very sparse and imprecise sensor information and outperforms a standard Monte Carlo localization approach.
\end{abstract}

\section{INTRODUCTION}

Recently, the robotics community has shown an increasing interest in small-sized and low-cost autonomous aerial vehicles such as helicopters, quadrotors, or blimps. Especially their low power consumption and safe navigation capabilities make blimps ideally suited for long-term indoor operation tasks. One of the most fundamental abilities of autonomously operating robots is to localize themselves in a known environment. This has been successfully addressed by Bayes filter techniques in the past [22]. The smaller a flying robot gets, however, the less sensor information is available due to their strictly limited number of lightweight and imprecise sensors. This increases the importance of the prediction model of the Bayes filter localization which is often also called the motion model of the robot. Most ground vehicles are equipped with wheel encoders and can sense their motion relative to the ground in a fairly accurate way. Motion models of aerial robots, however, can not rely on direct measurements of the velocity and in general need to estimate accelerations due to thrust and air drag. Consequently, the motion models are based on the complex kinematics of the vehicle which can be modeled by physical approximations and depends on numerous parameters. In practice, these parameters are usually hand-tuned by a human operator or derived from tedious calibration experiments using expert knowledge or ground-truth pose estimates.

This work has partly been supported by the German Research Foundation (DFG) within the Research Training Group 1103 and by the Gottfried Wilhelm Leibniz Programme of the DFG. All authors are members of the Department of Computer Science at the University of Freiburg, Germany

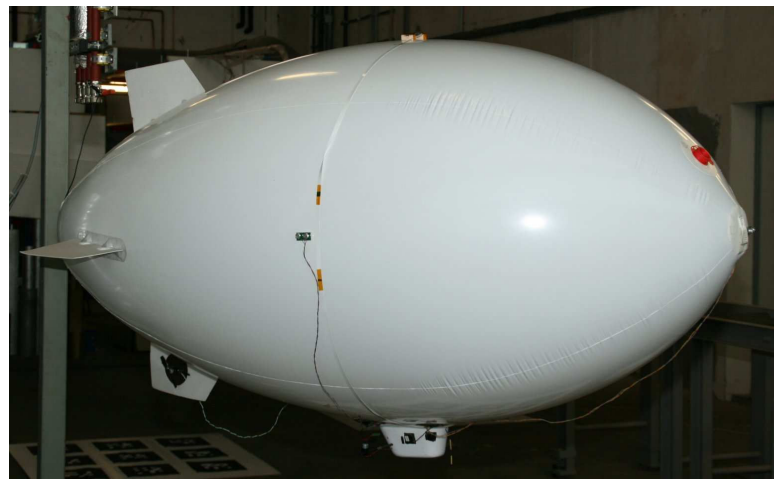

Fig. 1. The robotic indoor blimp [19] used throughout this paper. It is actuated by two main propellers that pivot together and a third laterally mounted rear propeller. Four small, lightweight wide-angle sonar sensors provide distance measurements to the environment.

In this paper, we consider the problem of localizing a small-size blimp in indoor environments using Monte Carlo localization with very sparse sensor information. Our blimp [19], which is depicted in Fig. 1, has an effective payload of 100 grams and is equipped with four miniature sonar sensors. Due to their huge opening angle the sonar sensors provide only few information about the orientation of the blimp. To cope with this lack of sensor information we improve the proposal distribution by simultaneously estimating the uncertain parameters of the motion model. Our approach allows for an online localization and has a couple of substantial advantages. It does not rely on previous calibration of motion model parameters and can adapt to changing parameters during operation. Our method explicitly includes a damping to prevent an overshooting of the parameters for situations, in which parameter changes can only be detected with certain delays. We envision a wide range of applications of our approach including nonconstant payload of the blimp, deformations of the blimp due to collisions, or even for ground robots, to deal with, for example, ground-dependent wheel slippage. Furthermore, the estimated parameters in combination with the underlying physical motion model can be used for an online adaption of motion controllers [18], [25] in autonomous navigation scenarios.

This paper is organized as follows. After discussing related work in the following section, we describe Monte Carlo localization with simultaneous parameter estimation in section III and discuss the adaption capabilities to changing parameters during operation. We will then briefly present a probabilistic motion model for robotic indoor blimps in 
section IV and finally evaluate the improvements of our localization system with simultaneous parameter estimation in simulation and experiments with a real blimp.

\section{RELATED WORK}

In the past, several authors considered the problem of localizing small flying vehicles. The majority of approaches, however, employed previously learned motion models. For example, Ko et al. [11] used the ground-truth estimates of a motion capture system for tuning of the motion model. Our previous work [15] relies on vision-based ground-truth and uses additional IMU sensor information for localization. Acquiring the ground-truth data involves either expensive and bulky systems or lacks sufficient precision to infer highly accurate motion models.

Several approaches have been proposed to improve the proposal distribution in Monte Carlo localization using information from sensors other than wheel encoders or control commands. For example, Thrun et al. [23] sample additional "dual" particles from the observation likelihood to improve the robustness of the system and to better recover from localization failures. Whereas this approach is very effective, sampling from the observation likelihood is computationally demanding for range sensors such as sonar or laser range finders. Consequently, sampling from the observation model was mainly used for vision-based localization. Grisetti et al. [6] matched laser range scans to improve proposals for wheeled robots operating on planar grounds. Later, a similar approach was applied together with IMU data to localize miniature quadrotors operating in 6 degrees of freedom (DOF) [7], [9]. However, it is unclear whether small lightweight sensors such as three miniature wide-angle sonar sensors allow for such a scan matching approach.

One of the first systems for Monte Carlo localization with online calibration of the motion model was developed by Roy and Thrun [20]. They incrementally update the calibration parameters for differential drive robots based on a maximum likelihood position estimate obtained by scan matching. However, their approach relies on the direct calculation of parameters out of two consecutive pose estimates which is not possible for more sophisticated motion models such as those for blimps. The case of sudden changes of the models or their parameters due to failures or collisions of wheeled robots is addressed by Plagemann et al. [16], [17]. They extend the particle filter localization by motion models of different complexity and use a parameter sampling similar to our approach. However, they assume an initially known model including its parameters and switch online between a finite number of models.

Beside the Monte Carlo localization, Kalman filters are a popular technique for mobile robot localization. For example, Martinelli et al. [14] extend the state vector by additional parameters for odometry errors. However, motion models of flying vehicles typically are highly non-linear and our wide-angle sonar sensors are not suitable to use within a Kalman filter. In the context of localization of UAVs, Bryson and Sukkarieh [2] estimate the difference between IMU and

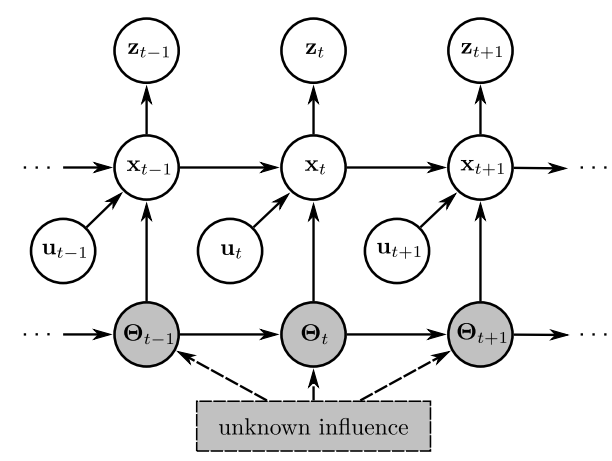

Fig. 2. The extended dynamic Bayes network for localization of a mobile robot. It characterizes the evolution of controls $\mathbf{u}$, states $\mathbf{x}$, measurements $\mathbf{z}$, and parameters of the motion model $\Theta$.

motion model prediction in an additional extended Kalman filter to update the model parameters and the IMU bias. In contrast to our approach this requires to update the model parameters directly based on the prediction error similar to Roy and Thrun [20].

Some approaches utilize the expectation maximization algorithm to simultaneously localize a robot and adapt its motion model. For example, Eliazar and Parr [5] iterate between estimating the path of the robot and optimizing the parameters of the motion model. However, these approaches are not intended for online applications and require to quickly optimize the parameters of the motion model given a trajectory of the robot which is not the case, e.g., for more complex motion models of flying robots such as blimps. Kaboli et al. [10] use the Markov Chain Monte Carlo technique to learn sensor and motion model parameters from raw sensor and action data by sampling trajectories and parameters, which typically requires substantial computational resources.

In contrast to these previous approaches, our approach provides an online estimation and adaptation of previously unknown parameters of a complex and potentially non-linear motion model in the context of localization with particle filters. Furthermore, compared to multiple model tracking systems our approach can deal better with very slow changes in the continuous parameter space.

\section{Monte Carlo Localization with SimUlTANEOUS PARAMETER ESTIMATION}

Throughout this paper, we consider the problem of estimating the 6-dimensional pose $\mathbf{x}=(x, y, z, \phi, \theta, \psi)$ of a robot relative to a given map $m$ using Monte Carlo localization [3]. The key idea of this approach is to maintain a probability density $p\left(\mathbf{x}_{1: t} \mid \mathbf{z}_{1: t}, \mathbf{u}_{1: t}\right)$ of the trajectory $\mathbf{x}_{1: t}$ of the robot given all observations $\mathbf{z}_{1: t}$ and control inputs $\mathbf{u}_{1: t}$ up to time $t$.

\section{A. Simultaneous Parameter Estimation}

In the presence of unknown (and probably time-varying) parameters of the motion of the robot, the underlying Bayes network is extended as depicted in Fig. 2. Here, the additional, non-observable parameter nodes are highlighted in gray. Consequently, the full localization posterior is extended 
to $p\left(\mathbf{x}_{1: t}, \boldsymbol{\Theta}_{1: t} \mid \mathbf{z}_{1: t}, \mathbf{u}_{1: t}\right)$ which can be factorized to

$$
\begin{aligned}
p\left(\mathbf{x}_{1: t}, \boldsymbol{\Theta}_{1: t} \mid \mathbf{z}_{1: t}, \mathbf{u}_{1: t}\right)= & \eta \cdot p\left(\mathbf{z}_{t} \mid \mathbf{x}_{1: t}, \mathbf{z}_{1: t-1}, \boldsymbol{\Theta}_{1: t}, \mathbf{u}_{1: t}\right) \\
& \cdot p\left(\mathbf{x}_{1: t}, \boldsymbol{\Theta}_{1: t} \mid \mathbf{z}_{1: t-1}, \mathbf{u}_{1: t}\right)
\end{aligned}
$$

using Bayes rule where $\eta$ is a normalizer. We factorize the second conditional probability twice and obtain

$$
\begin{aligned}
p\left(\mathbf{x}_{1: t}, \boldsymbol{\Theta}_{1: t} \mid\right. & \left.\mathbf{z}_{1: t-1}, \mathbf{u}_{1: t}\right) \\
= & p\left(\mathbf{x}_{t}, \boldsymbol{\Theta}_{t} \mid \mathbf{x}_{1: t-1}, \boldsymbol{\Theta}_{1: t-1}, \mathbf{z}_{1: t-1}, \mathbf{u}_{1: t}\right) \\
& \cdot p\left(\mathbf{x}_{1: t-1}, \boldsymbol{\Theta}_{1: t-1} \mid \mathbf{z}_{1: t-1}, \mathbf{u}_{1: t}\right) \\
= & p\left(\mathbf{x}_{t} \mid \mathbf{x}_{1: t-1}, \boldsymbol{\Theta}_{1: t}, \mathbf{z}_{1: t-1}, \mathbf{u}_{1: t}\right) \\
& \cdot p\left(\boldsymbol{\Theta}_{t} \mid \mathbf{x}_{1: t-1}, \boldsymbol{\Theta}_{1: t-1}, \mathbf{z}_{1: t-1}, \mathbf{u}_{1: t}\right) \\
& \cdot p\left(\mathbf{x}_{1: t-1}, \boldsymbol{\Theta}_{1: t-1} \mid \mathbf{z}_{1: t-1}, \mathbf{u}_{1: t}\right) .
\end{aligned}
$$

Under the Markov assumption, (1) together with (2) can be simplified to

$$
\begin{aligned}
p\left(\mathbf{x}_{1: t}, \boldsymbol{\Theta}_{1: t} \mid\right. & \left.\mathbf{z}_{1: t}, \mathbf{u}_{1: t}\right) \\
= & \eta \cdot p\left(\mathbf{z}_{t} \mid \mathbf{x}_{t}\right) \\
& \cdot p\left(\mathbf{x}_{t} \mid \mathbf{x}_{t-1}, \boldsymbol{\Theta}_{t}, \mathbf{u}_{t}\right) \\
& \cdot p\left(\boldsymbol{\Theta}_{t} \mid \boldsymbol{\Theta}_{t-1}, \mathbf{x}_{1: t-1}, \mathbf{z}_{1: t-1}\right) \\
& \cdot p\left(\mathbf{x}_{1: t-1}, \boldsymbol{\Theta}_{1: t-1} \mid \mathbf{z}_{1: t-1}, \mathbf{u}_{1: t-1}\right) .
\end{aligned}
$$

As in [22], we assume that future controls give no information about the current state of the robot.

To implement this recursive filtering scheme, we use a particle filter [4] where a set $\mathcal{M}$ of weighted particles represents the current belief. Each particle represents a hypothesis of a robot pose and parameter vector. As the proposal distribution we use the motion model combined with the model of the parameter behavior

$$
\begin{aligned}
\pi\left(\mathbf{x}_{1: t}, \boldsymbol{\Theta}_{1: t} \mid\right. & \left.\mathbf{z}_{1: t}, \mathbf{u}_{1: t}\right) \\
= & p\left(\mathbf{x}_{t} \mid \mathbf{x}_{t-1}, \boldsymbol{\Theta}_{t}, \mathbf{u}_{t}\right) \\
& \cdot p\left(\boldsymbol{\Theta}_{t} \mid \boldsymbol{\Theta}_{t-1}, \mathbf{x}_{1: t-1}, \mathbf{z}_{1: t-1}\right) \\
& \cdot p\left(\mathbf{x}_{1: t-1}, \boldsymbol{\Theta}_{1: t-1} \mid \mathbf{z}_{1: t-1}, \mathbf{u}_{1: t-1}\right)
\end{aligned}
$$

which results in the importance weight

$$
\begin{aligned}
w_{t}^{[i]} & =\frac{p\left(\mathbf{x}_{1: t}, \boldsymbol{\Theta}_{1: t} \mid \mathbf{z}_{1: t}, \mathbf{u}_{1: t}\right)}{\pi\left(\mathbf{x}_{1: t}, \boldsymbol{\Theta}_{1: t} \mid \mathbf{z}_{1: t}, \mathbf{u}_{1: t}\right)} \\
& \propto p\left(\mathbf{z}_{t} \mid \mathbf{x}_{t}\right) w_{t-1}^{[i]}
\end{aligned}
$$

of the $i$-th particle at time $t$.

Assuming the parameters to be constant over time, the belief update (3) can be performed according to the following three alternating steps:

1) In the prediction step, we draw for each particle a new particle according to the parameterized motion model $p\left(\mathbf{x}_{t} \mid \mathbf{x}_{t-1}, \mathbf{\Theta}_{t}, \mathbf{u}_{t}\right)$ given the action $\mathbf{u}_{t}$.

2) In the correction step, we integrate a new observation $\mathbf{z}_{t}$ by assigning a new weight $w^{[i]}$ to each particle according to the sensor model $p\left(\mathbf{z}_{t} \mid \mathbf{x}_{t}\right)$.

3) In the resampling step, we draw a new generation of particles from $\mathcal{M}$ (with replacement) such that each sample in $\mathcal{M}$ is selected with a probability that is proportional to its weight.
Following Liu and West [12], we reduce the sample degeneracy/attrition by adding small random disturbances to the parameter vector of each sample during resampling. To prevent a loss of information in the parameter vector sampling we apply kernel smoothing

$$
\boldsymbol{\Theta}_{t}^{[i]} \sim \mathcal{N}\left(a \Theta_{t-1}^{[i]}+(1-a) \overline{\boldsymbol{\Theta}}_{t-1}, h^{2} \mathbf{V}_{t-1}\right),
$$

where $\overline{\boldsymbol{\Theta}}_{t-1}$ and $\mathbf{V}_{t-1}$ are the mean and the covariance of the parameter vector over the particle set at time $t-1$. The constant factors $a=\frac{3 \gamma-1}{2 \gamma}$ and $h^{2}=1-a^{2}$ only depend on a discount factor $\gamma$, which we set to 0.95 .

\section{B. Adaption to Changed Parameters}

In certain cases, we cannot assume the physical properties of the motion of the robot to be constant for the complete period of operation. For example, wear and tear, changed payload, collisions, low batteries, or even manual mounting of banner ads can change the behavior of the robot. Once the parameter vector has converged within the particle filter, an adaption to one or more changed parameters would take a large number of sampling steps or could simply be impossible.

Fortunately, this problem can be solved in a similar way as the well-known "kidnapped robot" problem by sampling an appropriate number of the particles at random positions [22]. We analogously cope with parameter changes by drawing the parameter vector $\Theta$ uniformly from the parameter space for those random samples. The proportion of random samples is determined by monitoring the probability of sensor measurements $\bar{p}=\frac{1}{N} \sum_{i=1}^{N} p\left(\mathbf{z}_{t} \mid \mathbf{x}_{t}^{[i]}\right)$ averaged over all particles. We adopt this technique from Gutmann and Fox [8] and extend the resampling by setting the parameter vector of each particle to a uniform sample with probability

$$
\max \left(0,1-\frac{p_{\text {short }}}{\nu p_{\text {long }}}\right) .
$$

Here, $p_{\text {short }}$ and $p_{\text {long }}$ are short-term and long-term averages of the sensor likelihood updated by

$$
\begin{gathered}
p_{\text {short }} \leftarrow p_{\text {short }}+\alpha_{\text {short }}\left(\bar{p}-p_{\text {short }}\right) \\
p_{\text {long }} \leftarrow p_{\text {long }}+\alpha_{\text {long }}\left(\bar{p}-p_{\text {long }}\right)
\end{gathered}
$$

on each correction step with the exponential decay factors $0<\alpha_{\text {long }} \ll \alpha_{\text {short }} \leq 1$. The parameter $\nu$ allows to adjust the level at which random samples are added. Thus, this approach only adds random samples if the short-term average of the sensor likelihood is less than $\nu$ the long-term average.

However, the sole addition of random parameter samples does not yield superior results since the adaption to changed parameters does not start until the pose estimate is slightly displaced from the ground-truth caused by wrong parameters and the average observation likelihood is dropped. When random parameter samples are added, those particles which quickly correct the displacement will get a higher observation likelihood. This leads to an overshooting of the estimated parameter values and causes the parameter values to oscillate 
for several time steps (see Fig. 5, left). We address this oscillation problem by defining a lower bound to the covariance $h^{2} \mathbf{V}$, so that the parameter vectors are sampled according to

$$
\begin{aligned}
\boldsymbol{\Theta}_{t}^{[i]} \sim \mathcal{N}\left(a \boldsymbol{\Theta}_{t-1}^{[i]}+(1-a) \overline{\boldsymbol{\Theta}}_{t-1},\right. \\
\left.h^{2} \widetilde{\max }\left(\mathbf{V}_{t-1}, \rho \overline{\boldsymbol{\Theta}}_{t-1} \overline{\boldsymbol{\Theta}}_{t-1}^{T}\right)\right)
\end{aligned}
$$

where the $\widetilde{\max }$-operator builds a pointwise maximum over the diagonal elements of the matrices and takes all other elements from the first argument. Here, $\rho$ is the relative covariance bound. This damps the oscillation and allows for a lower localization error after the change of parameters. Furthermore, this approach better handles very slow changes of parameters which are typically not detected through a suddenly dropped observation likelihood.

Combining these techniques enables an autonomous robot to simultaneously estimate previously unknown parameters of the motion model in an online fashion and to adapt to changed parameters during operation, which is especially important in case of sparse or imprecise sensor information.

\section{Probabilistic Motion Models for Miniature AIRSHIPS}

The probabilistic motion model $p\left(\mathbf{x}_{t} \mid \mathbf{u}_{t}, \mathbf{x}_{t-1}\right)$ plays a crucial role in the prediction step of the particle filter and its proper design is essential for accurate and efficient state estimates. An inaccurate motion model would result in a wide-spread proposal distribution and increases the number of wasted particles which in turn decreases the efficiency of the filter. To define an accurate probabilistic model, we first create a deterministic physical model of our blimp and afterwards extend this model such that it also considers the sources of uncertainty.

Miniature airships typically are not equipped with sensors directly measuring their motion such as wheel encoders found on most ground vehicles. Instead, their motion has to be estimated based on forces and torques acting on them. In the following, we summarize our physical motion model which is based on [25]. The state of the blimp is defined by its pose $\mathbf{p}=(x, y, z, \phi, \theta, \psi)^{T}$ consisting of three Cartesian translation coordinates and three Euler angles. Accordingly, its velocity is $\mathbf{v}=\left(v_{x}, v_{y}, v_{z}, \omega_{x}, \omega_{y}, \omega_{z}\right)^{T}$. The NewtonEuler equation of motion

$$
M \frac{d \mathbf{v}}{d t}=\sum \mathbf{F}_{\text {external }}
$$

couples the acceleration of the airship to the external forces and torques. Thereby, $M$ is the rigid-body inertia matrix and the external forces are gravity, buoyancy, propelling forces, air drag forces, and fictitious forces. Except for the air drag forces, all constituent parts can be determined in a straightforward way [25]. In the case of our blimp (see Fig. 1) the hull cannot be approximated appropriately by a highly symmetrical ellipsoid. Furthermore, the rear fins have an additional stabilizing effect.

In general, two different types of air drag forces can be distinguished: viscous resistance and quadratic drag. Since our blimp operates at velocities exceeding $1 \frac{\mathrm{m}}{\mathrm{s}}$ having a
Reynolds number $R_{e} \approx 30,000$, we can safely reduce the drag formula to the quadratic part. Consequently, we approximate the drag force and torque of the hull in an uncoupled way as

$$
\begin{aligned}
\mathbf{F}_{\mathrm{D}, \mathrm{h}}=( & -D_{x} v_{x}\left|v_{x}\right|,-D_{y} v_{y}\left|v_{y}\right|,-D_{z} v_{z}\left|v_{z}\right|, \\
& \left.-D_{x}^{\prime} \omega_{x}\left|\omega_{x}\right|,-D_{y}^{\prime} \omega_{y}\left|\omega_{y}\right|,-D_{z}^{\prime} \omega_{z}\left|\omega_{z}\right|\right)^{T} .
\end{aligned}
$$

Analogously, the drag force of each fin acts parallel to its normal and scales with its area and the fin drag coefficient $D_{\mathrm{f}}$. Due to symmetry we can set $D_{y}=D_{z}, D_{y}^{\prime}=D_{z}^{\prime}$, and $D_{x}^{\prime} \approx 0$ so that the motion model depends on a vector of four unknown parameters $\Theta=\left[D_{x}, D_{z}, D_{z}^{\prime}, D_{\mathrm{f}}\right]$

In our first localization system [15], we used a blimp motion model with fixed parameters and learned those parameters from about $8 \mathrm{~min}$ recorded data of our blimp based on the reference trajectory $T^{*}$ which was acquired using the camera integrated in the gondola of the blimp [21]. To find the parameter vector $\hat{\boldsymbol{\Theta}}$ which minimizes the incremental prediction error of the motion model we evaluated 30,000 uniformly sampled values and started a gradient descent minimization routine from the ten best random parameter vectors. As all of them converged to the same $\hat{\boldsymbol{\Theta}}$, we expect this to be the global minimum. These previously estimated parameters $\hat{\boldsymbol{\Theta}}$ are not used for our proposed localization with simultaneous parameter estimation.

The uncertainty of the motion model described above has basically three sources of uncertainty. Firstly, the applied physical model is only an approximation, and secondly, the estimated parameters are not guaranteed to be the true parameters due to imperfection of the reference trajectory and the minimization routine. While both of these are systematic errors, thirdly, there are statistical errors such as imperfect motor responses, wind, or numerical errors. In our localization system, we combine all three sources of error and sample an additive velocity from a multivariate Gaussian with zero mean. Its covariance was determined based on the remaining error of the parameter optimization routine.

\section{EXPERIMENTAL EVALUATION}

The Monte Carlo localization with simultaneous parameter estimation described above has been implemented and evaluated using simulation experiments and data acquired with a real indoor blimp. In our experiments, we consider the problem of position tracking, i.e., localization with a known initial pose. To address the particle depletion problem, the resampling step is only performed if the number of effective particles [13] $n_{\text {eff }}=\left(\sum_{i}\left(w^{[i]}\right)^{2}\right)^{-1}$ drops below a threshold $\kappa$. In our experiments, this is set to half the number of particles. As a measure of localization error we used the Euclidean distance between the weighted average of all particles and the reference pose.

\section{A. Experiments with a real blimp}

We evaluated the improvement of the simultaneous parameter estimation in terms of localization error using a $1.70 \mathrm{~m}$ long blimp [19] in a large indoor environment. The blimp is actuated by two main propellers that pivot 


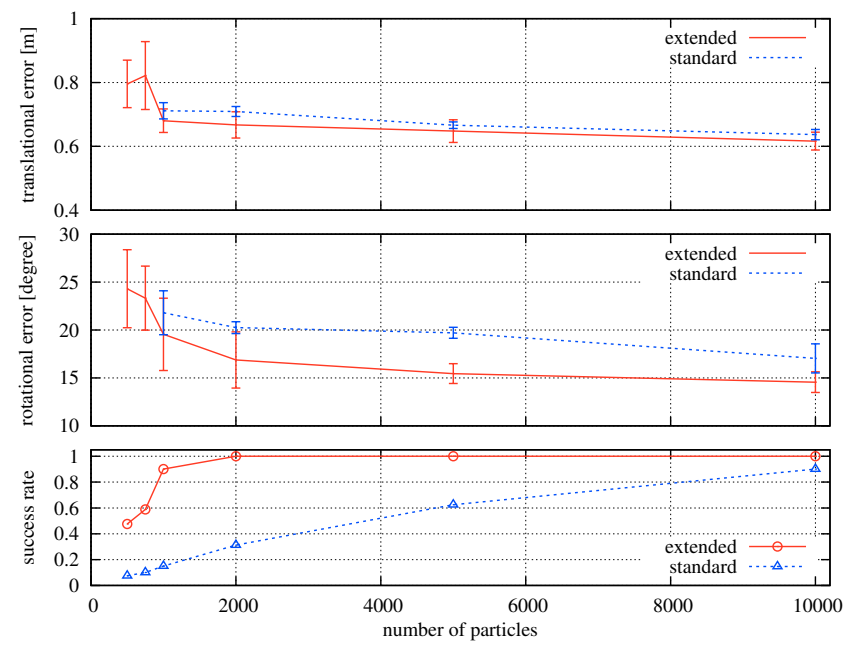

Fig. 3. The average RMS localization error and the success rate of the extended Monte Carlo localization with simultaneous parameter estimation compared to the standard localization with previously learned parameters of the motion model. The error bars indicate the $5 \%$ confidence intervals over ten runs.

together and a third laterally mounted rear propeller for yaw rotation. Four small, lightweight wide-angle sonar sensors provide distance measurements to the environment, which are probabilistically modeled as described in our previous work [15]. The experimental indoor environment has an area for flying of about $14 \times 7 \mathrm{~m}^{2}$ with a vertical space of $5 \mathrm{~m}$ and was mapped from 3D laser scans using multi-level surface maps [24]. To determine the localization error, we put visual markers onto the floor, which allow us to accurately calculate the pose of the vehicle using the camera integrated in the gondola of the blimp [1].

In an extensive experiment of about 9 minutes of manually operated flight the blimp covered a distance of about $180 \mathrm{~m}$. Since we did not use an IMU and the wide-angle sonar sensors provide hardly any information about the orientation of the blimp, the localization relies on an accurate prediction of the motion model. As can be seen in Fig. 3, the Monte Carlo localization with simultaneous estimation of initially unknown parameters benefits from the improved proposal distribution. It resulted in a significantly lower rotational localization error compared to the results obtained using the implementation with previously learned (see section IV) and fixed parameters of the motion model. The estimate of the parameter vector typically converged within the first minute of the experiment. Although the dimensionality of the state estimation problem was increased by the four parameters, the localization success rate revealed that the number of particles required to reliably localize the blimp is substantially smaller using the simultaneous parameter estimation.

Furthermore, we tested our localization system with simultaneous parameter estimation in a global localization szenario with initially unknown pose and motion model parameters. We carried out 10 runs with 5,000 particles each. Whereas the pose estimate typically converged within the first 10 seconds, it still took 1 minute to determine the parameters. Based on these experiments we expect that

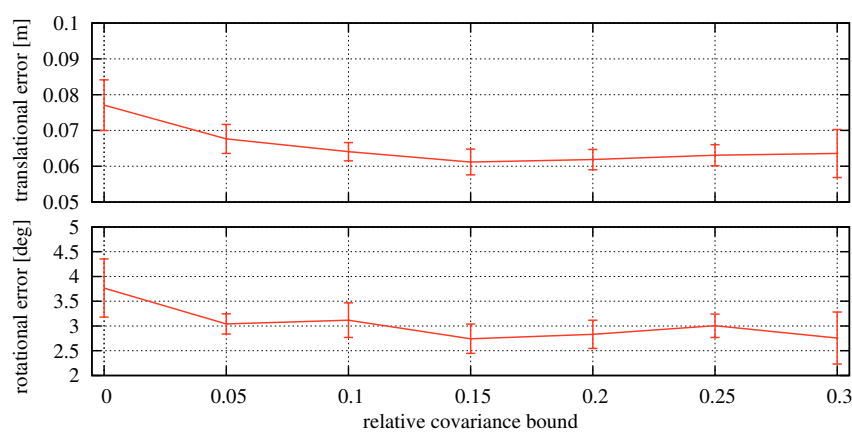

Fig. 4. The average translational and rotational RMS localization error of a 10 minute localization experiment on simulation data. The error bars indicate the $5 \%$ confidence intervals over ten runs.

our approach can also handle the kidnapped robot problem given an extension which can distinguish between parameter changes and kidnappings based on drops of the observation likelihood.

\section{B. Simulation of Changing Parameters}

To evaluate the capability of our localization system to adapt to changing parameters of the motion model, we performed a series of experiments on a simulated blimp using our deterministic motion model. The true poses were passed as observations to the localization system and their likelihood were modeled as a Gaussian distribution with high translational and low rotational precision $\left(\sigma_{\text {trans }}=0.2 \mathrm{~m}\right.$, $\sigma_{\text {rot }}=15^{\circ}$ ).

In an experiment of about 10 minutes of manually operated flight we evaluated different relative covariance bounds $\rho$ in our localization system using 5,000 particles. Note that we experimentally tuned the parameters $\alpha_{\text {short }}=0.2, \alpha_{\text {long }}=$ 0.01 , and $\nu=0.8$ to obtain best results. Although we introduced only little noise to the velocity of the blimp during simulation, the parameter estimation during localization was a challenging task due to the correlation of the different parameters (see Fig. 5). Furthermore, estimating all parameters requires a certain spectrum of movements. For example, during the first seconds of our experiment the blimp was controlled to move forward only which resulted in a quick convergence of solely the drag coefficient $D_{x}$.

Fig. 4 presents the localization accuracy on the simulated data during which the parameters of the motion model were changed as depicted in Fig. 5. Both, the translational and rotational localization error are significantly lower for a relative covariance bound of $\rho>0.15$ than without a lower bound $(\rho=0)$ of the covariance of the parameters.

\section{Vi. CONCLUSIONS}

In this paper, we presented a novel approach to Monte Carlo localization of autonomous robots with simultaneous estimation of the parameters of the motion model. In contrast to other approaches, our systems allows an online localization without prior knowledge of all motion model parameters and can adapt to changed parameters during operation. To avoid oscillations after parameter changes on systems, for which these changes can only be detected with a 

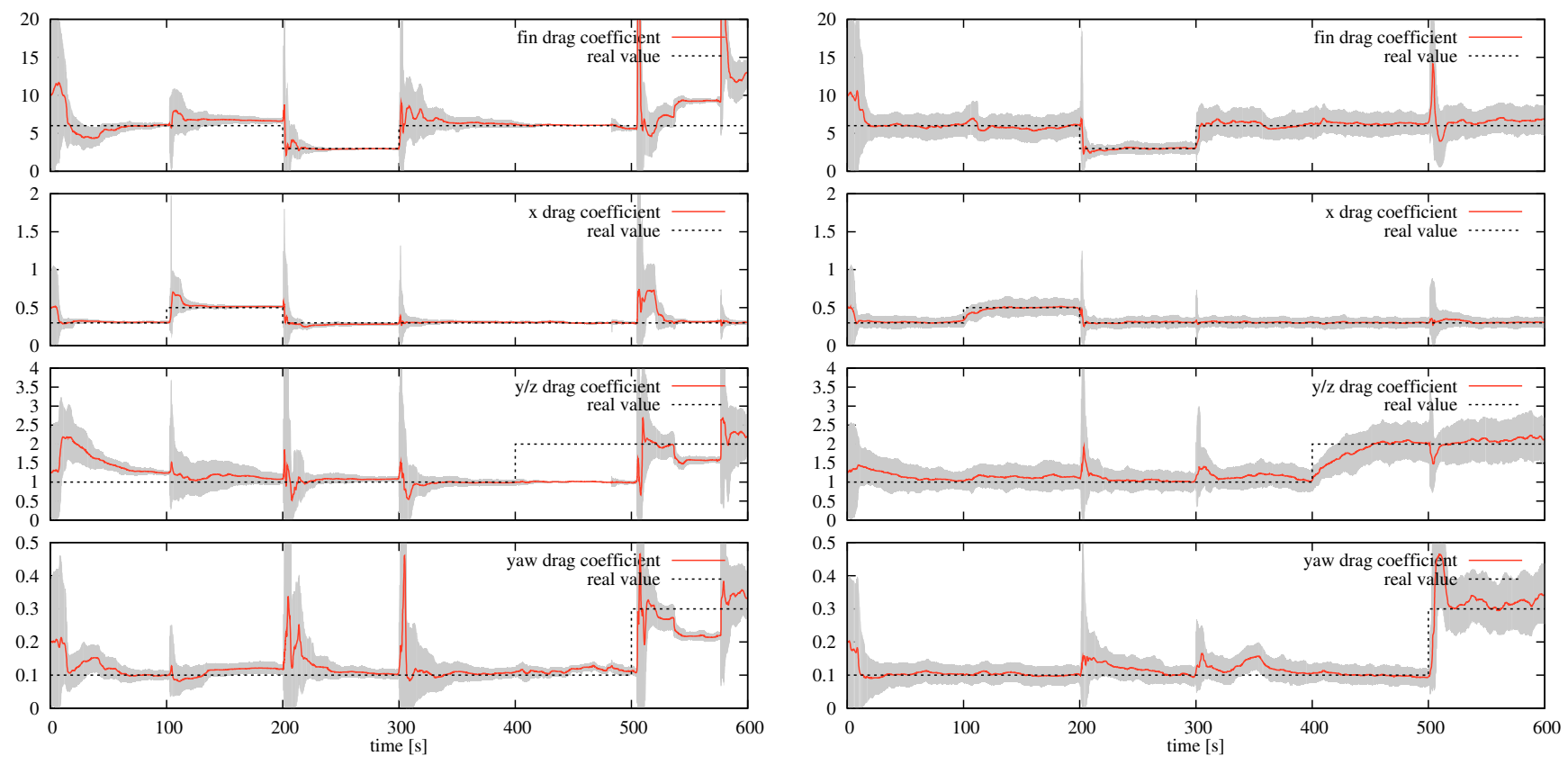

Fig. 5. The parameter estimation of exemplary localization runs of the simulated blimp without covariance bounding (left) and with $\rho=0.2$ (right). The area between the $2.5 \%$ and $97.5 \%$ quantiles of the estimated posterior is marked gray.

certain delay, our approach includes an appropriate damping mechanism. In experiments carried out with a real blimp and in simulation, we demonstrated that our system significantly outperforms the standard Monte Carlo localization with previously learned parameters in terms of the localization accuracy and the number of particles needed.

\section{REFERENCES}

[1] M. Billinghurst and H. Kato. Collaborative augmented reality. Communications of the ACM, (7), 2002.

[2] M. Bryson and S. Sukkarieh. Vehicle model aided inertial navigation for a UAV using low-cost sensors. In Proc. of the Australasian Conf. on Robotics \& Automation (ACRA), 2004.

[3] F. Dellaert, D. Fox, W. Burgard, and W. Thrun. Monte carlo localization for mobile robots. In Proc. of the IEEE Int. Conf. on Robotics \& Automation (ICRA), 1999.

[4] A. Doucet. On sequential simulation-based methods for bayesian filtering. Technical report, Signal Processing Group, Dept. of Engeneering, University of Cambridge, 1998.

[5] A.I. Eliazar and R. Parr. Learning probabilistic motion models for mobile robots. In Proc. of the Int. Conf. on Machine Learning, 2004.

[6] G. Grisetti, C. Stachniss, and W. Burgard. Improving grid-based slam with rao-blackwellized particle filters by adaptive proposals and selective resampling. In Proc. of the IEEE Int. Conf. on Robotics \& Automation (ICRA), 2005.

[7] S. Grzonka, G. Grisetti, and W. Burgard. Towards a navigation system for autonomous indoor flying. In Proc. of the IEEE Int. Conf. on Robotics \& Automation (ICRA), 2009.

[8] J.-S. Gutmann and D. Fox. An experimental comparison of localization methods continued. In Proc. of the IEEE/RSJ Int. Conf. on Intelligent Robots and Systems (IROS), Lausanne, Switzerland, 2002.

[9] R. He, S. Prentice, and N. Roy. Planning in information space for a quadrotor helicopter in a gps-denied environments. In Proc. of the IEEE Int. Conf. on Robotics \& Automation (ICRA), 2008.

[10] A. Kaboli, M. Bowling, and P. Musilek. Bayesian calibration for monte carlo localization. In Proc. of the National Conf. on Artificial Intelligence (AAAI), 2006.

[11] J. Ko, D.J. Klein, D. Fox, and D. Haehnel. GP-UKF: Unscented kalman filters with gaussian process prediction and observation models. In Proc. of the IEEE/RSJ Int. Conf. on Intelligent Robots and Systems (IROS), 2007.
[12] J. Liu and M. West. Combined parameter and state estimation in simulation-based filtering. In Arnaud Doucet, Nando de Freitas, and Neil Gordon, editors, Sequential Monte Carlo Methods in Practice, chapter 10. 2001.

[13] J.S. Liu. Metropolized independent sampling with comparisons to rejection sampling and importance sampling. Statist. Comput., 6, 1996.

[14] A. Martinelli, N. Tomatis, A. Tapus, and R. Siegwart. Simultaneous localization and odometry calibration for mobile robot. In Proc. of the IEEE/RSJ Int. Conf. on Intelligent Robots and Systems (IROS), 2003.

[15] J. Müller, A. Rottmann, and W. Burgard. A probabilistic sonar sensor model for robust localization of a small-size blimp in indoor environments using a particle filter. In Proc. of the IEEE Int. Conf. on Robotics \& Automation (ICRA), 2009.

[16] C. Plagemann and W. Burgard. Sequential parameter estimation for fault diagnosis in mobile robots using particle filters. In Autonome Mobile Systeme 2005 (AMS). Springer, 2005.

[17] C. Plagemann, C. Stachniss, and W. Burgard. Efficient failure detection for mobile robots using mixed-abstraction particle filters. In H.I. Christensen, editor, European Robotics Symposium 2006, volume 22 of STAR Springer tracts in advanced robotics. Springer Verlag, 2006.

[18] A. Rottmann, C. Plagemann, P Hilgers, and W. Burgard. Autonomous blimp control using model-free reinforcement learning in a continuous state and action space. In Proc. of the IEEE/RSJ Int. Conf. on Intelligent Robots and Systems (IROS), 2007.

[19] A. Rottmann, M. Sippel, T. Ziterell, W. Burgard, L. Reindl, and C. Scholl. Towards an experimental autonomous blimp platform. In Proc. of the European Conf. on Mobile Robots (ECMR), 2007.

[20] N. Roy and S. Thrun. Online self-calibration for mobile robots. In Proc. of the IEEE Int. Conf. on Robotics \& Automation (ICRA), 1999.

[21] B. Steder, G. Grisetti, S. Grzonka, C. Stachniss, A. Rottmann, and W. Burgard. Learning maps in $3 \mathrm{~d}$ using attitude and noisy vision sensors. In Proc. of the IEEE/RSJ Int. Conf. on Intelligent Robots and Systems (IROS), 2007.

[22] S. Thrun, W. Burgard, and D. Fox. Probabilistic Robotics. MIT Press, 2005.

[23] S. Thrun, D. Fox, and W. Burgard. Monte carlo localization with mixture proposal distribution. In Proc. of the National Conf. on Artificial Intelligence (AAAI), 2000.

[24] R. Triebel, P. Pfaff, and W. Burgard. Multi-level surface maps for outdoor terrain mapping and loop closing. In Proc. of the IEEE/RSJ Int. Conf. on Intelligent Robots and Systems (IROS), 2006.

[25] J.C. Zufferey, A. Guanella, A. Beyeler, and D. Floreano. Flying over the reality gap: From simulated to real indoor airships. Autonomous Robots, 21(3), 2006. 